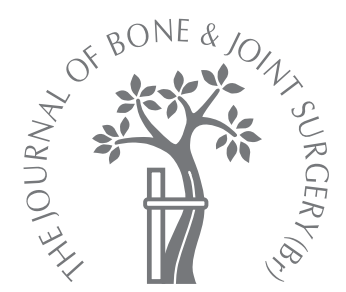

\title{
Long-term outcome of a transglenoid suture technique for anterior shoulder instability in young adults
}

T. Söderlund,

V. M. Mattila, T. I. Visuri, H. K. Pihlajamäki

\section{From the Central} Military Hospital and Centre for Military Medicine, Helsinki, Finland
- T. Söderlund, MD, PhD, Resident in Orthopaedic Surgery

Töölö Hospital, Helsinki University Central Hospital, PO Box 266, Helsinki, FIN-0029, Finland.

V. M. Mattila, MD, PhD, Resident in Orthopaedic Surgery

T. I. Visuri, MD, PhD, Adjunct Professor, Orthopaedic

Surgeon

H. K. Pihlajamäki, MD, PhD, Adjunct Professor, Orthopaedic Surgeon

Centre for Military Medicine, PO Box 50, FIN-00301 Helsinki, Finland.

Correspondence should be sent to Dr H. K. Pihlajamäki;

e-mail:

harri.pihlajamaki@helsinki.fi

(C2008 British Editorial Society of Bone and Joint Surgery doi:10.1302/0301-620X.90B2. $20040 \$ 2.00$

$J$ Bone Joint Surg $[B r]$ 2008;90-B:189-93.

Received 2 August 2007: Accepted 19 October 2007

\begin{abstract}
We reviewed the outcome of arthroscopic stabilisation of anterior glenohumeral instability in young adults using the transglenoid suture technique. A questionnaire was sent to 455 consecutive patients who had undergone this procedure between 1992 and 2000 . Of these, 312 patients $(68.5 \%)$ with 313 affected shoulders and a mean age of 20 years (18 to 28 ) responded.

Outcome was determined by the number of re-dislocations or, in patients who had not re-dislocated, by the disease-specific quality of life as measured by the Western Ontario Shoulder Instability index. During a mean follow-up of 6.4 years (1 to 14), 177 patients (56\%) sustained a re-dislocation, including 70 who required a further operation. In 136 patients $(44 \%)$ who reported neither re-dislocation nor re-operation, the index scores were good (median $\mathbf{9 0 . 4 \% ;} \mathbf{2 8 . 9 \%}$ to $100 \%$ ). No significant peri- or pre-operative predictors of redislocation or re-operation were found.

We found a high rate of re-dislocation after transglenoid suture repair in young, physically active patients.
\end{abstract}

Traumatic anterior dislocation of the shoulder is a common injury. Its lifetime prevalence is estimated to be $2 \%$ in the general population $^{1}$ and $7 \%$ among athletes. ${ }^{2}$ The risk of recurrent dislocation after primary trauma in individuals under 30 years of age has ranged from $26 \%$ to $90 \%$ after non-operative treatment ${ }^{3-5}$ and from $13 \%$ to $50 \%$ after operative treatment. ${ }^{5-8}$ The common findings at arthroscopy of the glenohumeral joint after the first anterior dislocation include haemarthrosis, a Hill-Sachs lesion, a Bankart lesion, a bony Bankart lesion, loose bodies, synovitis, a rotator cuff tear, humeral avulsion of the inferior glenohumeral ligament and a capsular tear. ${ }^{5,9,10}$

Various methods of surgical treatment are available, which include both open and arthroscopic techniques. Most of these focus on repair of the anterior capsulolabral complex, which is the principal passive anterior stabiliser of the shoulder. ${ }^{11}$ An arthroscopic transglenoid suture technique, also known as the Caspari technique, has been used since 1988. ${ }^{12}$ The rate of recurrence of dislocation after operative treatment by this method ranges between $0 \%$ and $49 \% .{ }^{13}$ Previous studies of this technique have usually included only a small number of patients followed up for a short time. The few studies with a follow-up of more than five years had small numbers of patients. ${ }^{6,8}$ Transglenoid suture repair allows tightening of the anterior capsule and re-attachment of the capsulolabral complex. However, the technique has received criticism because the capsulolabral complex may be attached too medially to the glenoid rim instead of in its normal anatomical location, ${ }^{6}$ which may lead to a reduction in the effective depth of the glenohumeral cavity. ${ }^{14}$ As the infraspinatus muscle is expanded by irrigation fluid during arthroscopy, there is a risk of loose fixation of the sutures. $^{15}$ Furthermore, drilling a hole through the neck of the scapula may damage the suprascapular nerve, in between $1.5 \%$ and $6 \%$ of cases. ${ }^{7,16}$

A recent meta-analysis investigating whether arthroscopic stabilisation was as effective as open surgery suggested that the failure rate using transglenoid sutures was higher than that using either open surgery or arthroscopic stabilisation using suture anchors or bioabsorbable tacks. ${ }^{17}$

The aim of this study was to assess the outcome of the transglenoid suture technique for anterior glenohumeral instability in a young adult population based on the recurrence of dislocation and the number of re-operations undertaken. 


\section{Patients and Methods}

In Finland, military service is compulsory for all men, but voluntary for women. Over $80 \%$ of men with a mean age of 20 years (18 to 29) complete a period of service of six to 12 months. ${ }^{18}$ Between 1992 and 2000 the number of conscripts starting their service each year varied between 20600 and 30500 .

We studied 455 consecutive military conscripts (449 men, 6 women) who underwent the transglenoid suture technique for first-time or recurrent anterior glenohumeral instability. After emergency care at a civilian hospital, these patients are always transferred to a military hospital. They were identified by a search of the medical records of the hospital between 1992 and 2000, using the appropriate diagnostic codes from the ninth and tenth revisions of the International Classification of Diseases. ${ }^{19}$ The inclusion criteria were a traumatic anterior dislocation of the shoulder and/or a posttraumatic recurrent dislocation stabilised arthroscopically using the transglenoid suture technique. Patients with a posterior dislocation, multidirectional instability, a previous stabilising operation on the same shoulder or operation for reasons other than instability were excluded. The study was approved by the Medical Ethics Committee of our hospital.

All 455 patients who met the inclusion criteria and underwent surgery between 1992 and 2000 were sent a questionnaire in 2005. Three further questionnaires were sent to nonrespondents. Altogether, 312 patients with 313 operated shoulders responded, a response rate of $68.5 \%$. At operation 308 patients $(98.4 \%)$ were male with a mean age of 20 years (18 to 28). The most common cause of the initial shoulder dislocation was a sports injury which occurred in 302 patients $(97 \%)$.

The outcomes assessed were recurrent anterior dislocation and the number of re-operations due to glenohumeral instability during follow-up. Both were thought to be due to failure of the primary operation. In patients who had not redislocated or required a further operation the diseasespecific quality of life was measured using the Western Ontario Shoulder Instability Index, ${ }^{1}$ which is a reliable method of assessing patients with either anterior or posterior instability. ${ }^{20}$ It measures four domains: physical symptoms, and pain, sports, recreation and work function, lifestyle and social function, and emotional well-being. A $100 \mathrm{~mm}$ visual analogue scale (VAS) where $0=$ no pain and $100=$ the worst pain and/or disability was used to determine the degree of pain and/or disability experienced by patients in the two weeks before answering the questionnaire. The results were used as outcome variables to describe the function of the operated shoulder. After subtracting the sum score for each patient from 2100 (21 variables each with maximum value of 100) the resulting value was given as a percentage with a higher percentage indicating a better outcome. Patients were questioned about dislocations before and after surgery, reoperations and treatment in other hospitals after their military service. The appropriate medical records were retrieved for review and analysis.
Table I. Characteristics of the patients at the time of surgery

\begin{tabular}{lr}
\hline Characteristics & Mean (range) \\
\hline Age in yrs (mean, range) & $20 \quad(18$ to 28) \\
Height in cm (mean, range) & 179 (162 to 197) \\
Weight in kg (mean, range) & 77 (55 to 120) \\
& \\
Number of dislocations before operative treatment & \\
(\%) & $154(49)$ \\
1 to 5 & $28(9)$ \\
6 to 10 & $102(33)$ \\
11 to 20 & $2(1)$ \\
$>20$ & $27(8)$ \\
$\quad$ Missing & $272(87)$ \\
Bankart lesion $\mathrm{n}(\%)$ & $300(96)$ \\
Hill-Sachs lesion $\mathrm{n}(\%)$ &
\end{tabular}

Follow-up continued until either re-dislocation, reoperation for glenohumeral instability or 31 December 2005. Re-operation was defined as further surgery after redislocation of the same shoulder. When asked about the year of dislocation, 81 patients $(26 \%)$ were unable to recall the event.

Table I gives the characteristics of the patients at the time of surgery. The median time between initial dislocation and surgery was 17 months ( 0 to 200). The right shoulder was involved in 164 cases.

Operative technique. Each operation was performed by one of five senior orthopaedic surgeons (TIV, HKP, OK, V-MN, $\mathrm{PJ})$ using the arthroscopic transglenoid method of suture repair described by Caspari. ${ }^{12}$ Under general anaesthesia, the patient was placed in the lateral decubitus position with traction applied to the arm. The posterior portal was used for the arthroscope and the anterior portal for instrumentation. After intra-articular examination and removal of any loose bodies the anteroinferior rim of the glenoid was abraded to expose cancellous bone at the site of reattachment, and a hole was drilled through the neck of the scapula between the 1 and 2 o'clock position for the right shoulder or the 10 and 11 o'clock position for the left. The inferior capsule was detached as necessary. Between three and eight polydioxanone sutures were passed through the labrum and the anterior capsule and then through the drill hole into the posterior scapula. At this stage the traction was released, the sutures were tightened, and the position of the labrum assessed. Knots were tied over the fascia on the dorsal side.

A sling was used for two to four weeks, after which the patients began physiotherapy. The shoulder was gradually mobilised, with a return to all sport activities within three months.

We then determined the number of re-dislocations and reoperations due to glenohumeral instability, analysed the predictors of re-dislocation or re-operation, and evaluated the outcome of the operation.

For statistical analysis, the $t$-test was used for continuous unskewed data, the Mann-Whitney U test for skewed contin- 
Table II. Predictors of poor outcome after surgery using a transglenoid suture technique

\begin{tabular}{|c|c|c|c|}
\hline & \multirow{2}{*}{$\frac{\text { Shoulders with re-dislocation or re-operation (\%) }}{\text { Number of shoulders }(\%)}$} & \multirow{2}{*}{$\begin{array}{l}\text { Shoulders without re-dislocation or re-operation (\%) } \\
\text { Number of shoulders }(\%)\end{array}$} & \multirow{2}{*}{ p-value* } \\
\hline & & & \\
\hline \multicolumn{4}{|l|}{ General laxity } \\
\hline No & $135(78.9)$ & $117(86.7)$ & 0.12 \\
\hline Yes & $36(21.1)$ & $18(13.3)$ & \\
\hline \multicolumn{4}{|l|}{$\operatorname{IUA}^{\dagger}$} \\
\hline None & $100(71.9)$ & $104(80.6)$ & 0.32 \\
\hline Subluxation & $24(17.3)$ & $18(14.0)$ & \\
\hline Luxation & $11(7.9)$ & $5(3.9)$ & \\
\hline \multicolumn{4}{|l|}{ Number of sutures } \\
\hline 1 & $0(0)$ & $1(0.8)$ & 0.60 \\
\hline 2 & $2(1.4)$ & $2(1.5)$ & \\
\hline 3 & $9(6.3)$ & $7(5.3)$ & \\
\hline 4 & $40(27.8)$ & $25(19.1)$ & \\
\hline 5 & $46(31.9)$ & $51(38.9)$ & \\
\hline 6 & $30(20.8)$ & $24(18.3)$ & \\
\hline 7 & $14(9.7)$ & $18(13.7)$ & \\
\hline 8 & $3(2.1)$ & $3(2.3)$ & \\
\hline \multicolumn{4}{|l|}{ Number of dislocations } \\
\hline 1 to 5 & $74(53.6)$ & $66(54.1)$ & 0.50 \\
\hline 6 to 10 & $13(9.4)$ & $12(9.8)$ & \\
\hline 11 to 20 & $51(37.0)$ & $42(34.4)$ & \\
\hline$>20$ & $0(0)$ & $2(1.6)$ & \\
\hline \multicolumn{4}{|l|}{ Contralateral shoulder laxity } \\
\hline No & $115(76.2)$ & $117(86.7)$ & 0.023 \\
\hline Yes & $36(23.8)$ & $18(13.3)$ & \\
\hline Delay to surgery in yrs (mean) & $1.9(0$ to 17$)$ & $1.9(0$ to 15$)$ & 0.68 \\
\hline
\end{tabular}

* the $p$-values refer to the differences between the two groups. Chi-squared test

† IUA, instability under anaesthesia

uous data, and the Kruskal-Wallis test for skewed continuous data between more than two groups. Correlation coefficients were calculated to determine the degree of association between continuous variables. The statistical difference between two independent groups was set to $\mathrm{p}=0.05$. (SPSS Statistical Software, SPSS Inc., Chicago, Illinois) was used. The chi-squared test was used with two-way tables.

\section{Results}

After a mean follow-up of 6.4 years (1 to 14), 177 $(56.5 \%)$ shoulders had a poor outcome and $136(43.5 \%)$ a good outcome with neither re-dislocation nor re-operation. The poor outcomes comprised 107 (34.2\%) re-dislocations and $70(22.4 \%)$ re-operations for instability. Of these latter patients, two underwent further surgery on three occasions, six on two occasions and 62 once. The redislocations resulted from sports or falling from a height in 41 patients $(38 \%)$, and from a sudden movement of the shoulder in $50(47 \%)$. In 16 patients $(15 \%)$ the re-dislocation had been non-traumatic, such as during sleep.

The median time from primary operation to re-dislocation was three years ( 1 to 13 ), and to re-operation 3.5 years (1 to 14$)$. Of the re-operations, 22 were carried out within a year of the original procedure.
The association between several variables and re-dislocation was analysed (Table II). No relationship was found between re-dislocation during follow-up and the operating surgeon ( $\mathrm{p}=0.41$, chi-squared test). None of the pathological findings noted during arthroscopy correlated with re-dislocation.

The outcome of surgery was analysed only in the 136 shoulders without re-dislocation or re-operation. The median Western Ontario Shoulder Instability index score was $90.4 \%(28.9 \%$ to $100 \%)$. There was no statistical difference in the results of the Western Ontario Shoulder Instability index scores for the five operating surgeons $(\mathrm{p}=$ 0.62 , Kruskal-Wallis), and no correlation ( $\mathrm{r}=-0.043$ ) between the time from first dislocation to repair and residual symptoms ( $\mathrm{p}=0.67$, correlation test). The pathological findings revealed during arthroscopy did not correlate with the Western Ontario Shoulder Instability index outcome.

The number of pre-operative dislocations was not associated with residual symptoms in patients who had not sustained a dislocation during the follow-up period ( $\mathrm{p}=$ 0.94, Kruskal-Wallis). However, dislocation in the contralateral shoulder was associated with an increase in postoperative symptoms (median Western Ontario Shoulder Instability score $79 \%(57 \%$ to $99 \%) ; p=0.02$, MannWhitney U test). Although there was a reduction in symp- 
toms with increasing length of follow-up, it did not reach statistical significance ( $p=0.54$, Mann-Whitney $U$ test). There was no difference in outcome between patients who underwent surgery within three months of the initial dislocation and those whose surgery was delayed for more than three months ( $\mathrm{p}=0.42$, Mann-Whitney U test).

There were $30(9.6 \%)$ minor post-operative complications: $27(8.6 \%)$ local skin irritations and/or suture infections and three $(0.9 \%)$ transient nerve lesions. All complications resolved eventually unremarkably with conservative treatment. The only intra-operative complication was a rupture of one or more sutures in 13 patients $(4.1 \%)$.

\section{Discussion}

Traumatic anterior dislocation of the shoulder is a common injury in young adults. The rate of recurrence is up to $100 \%$ in those treated conservatively. ${ }^{21}$ It has been suggested that young patients should undergo a stabilisation operation after the first traumatic dislocation. ${ }^{10,22}$ Some risk factors for the development of recurrent instability after arthroscopic stabilisation using suture anchors have recently been proposed as an instability severity index score. $^{23}$

We have investigated the outcome of the transglenoidal suture technique in young active patients. Because the data for recurrence were based on patient questionnaires, their validity can be questioned, but as all had suffered at least one anterior dislocation before the arthroscopic repair, they could be expected to recognise a recurrent dislocation.

The number of re-operations depended on the symptoms experienced after re-dislocation, the expectations of the patient and the availability of surgery. Overall, we considered the rate of recurrent dislocation to be a better indication of the outcome of operative treatment than the rate of re-operation.

Recurrent dislocation or instability occurred in 177 patients $(57 \%)$. Previous reports of this technique have described recurrence in between $0 \%$ and $49 \%$. $^{6-}$ $8,13,16,24,25$ Our study has the largest number of patients and longest follow-up reported so far, and shows that the rate of re-dislocation increases with time. This was also suggested by a recent retrospective study which compared suture anchors with transglenoidal sutures. ${ }^{24}$

There was no association between the intra-operative findings and the risk of re-dislocation. Although the size of the lesion of the glenoid labrum could be correlated with the rate of re-dislocation after transglenoid suture repair in a previous study, ${ }^{26}$ no such finding was seen in our study. Previous evidence indicating that the age of the patient at the time of operation is related to the rate of re-dislocation, with the highest rates observed in patients younger than 22 years, ${ }^{6,7,25}$ was confirmed by our study, where the mean age of the patients was 21.6 years.
The number of polydioxonone sutures used did not correlate with a poor outcome. An experimental study in rabbits with arthritic knees has shown that polydioxanone sutures may lose $60 \%$ of their initial strength within three weeks owing to the presence of inflammatory synovial fluids. ${ }^{27}$ If the initial transglenoidal sutures become loose because of the expansion of the infraspinatus muscle with irrigation fluid during arthroscopy, ${ }^{15}$ or if they lose more than half of their initial tensile strength over three weeks, the labral attachment will be relatively weak at the time of mobilisation of the shoulder. Kagaya et $\mathrm{al}^{28}$ compared absorbable sutures to absorbable plus non-absorbable sutures in the transglenoid technique. Recurrence of dislocation was higher when only absorbable sutures were used (26\% vs 6\%). ${ }^{28}$ Moreover, the effective depth, i.e., the distance from the most lateral extent of the anterior labrum to the medial extent of the glenoid cavity, was smaller after transglenoid suture repair than when using the suture anchor method. ${ }^{14}$

Of the re-dislocations in our study, 40 (37\%) occurred during sport by the same mechanism that caused the original dislocation. However, in other studies using different repair techniques the re-dislocation rates were lower, even though the patients continued to participate in diverse activities, including both collision and contact sports. ${ }^{29,30}$ It seems evident that the re-dislocations sustained by our patients during sports were the result of a repair that was weaker than that achieved with other techniques. The only factor in our study associated with re-dislocation was the occurrence of dislocation of the contralateral shoulder, a finding that suggests general laxity in these patients.

The subjective outcome was assessed using the Western Ontario Shoulder Instability index questionnaire in those patients who did not re-dislocate. None of the pre-operative or intra-operative variables studied showed any correlation with the Western Ontario Shoulder Instability index scores. The median Western Ontario Shoulder Instability index score was $90.4 \%$, which is similar to that found by Kirkley et $\mathrm{al}^{8}$ using the transglenoidal suture technique for repair after the initial dislocation. Shoulder function was good in patients who did not re-dislocate. When comparing Western Ontario Shoulder Instability index scores after the repair in our study with those seen after non-operative treatment in a prospective, randomised study, ${ }^{8}$ the scores were higher in the operativelytreated group ( $90.4 \%$ vs $74.8 \%$ ).

Our results indicate that the risk of redislocation is substantial if anterior glenohumeral instability is treated by transglenoid suture in young adults. The subjective outcome is good if re-dislocation does not occur and the risk of other complications is low. We agree with Mologne et $\mathrm{al}^{16}$ that this technique should not be recommended for young athletic patients with glenohumeral instability.

We would like to acknowledge Olli Kiviluoto, Veli-Matti Nurmi, Pekka Jokio, orthopaedic surgeons from the Central Military Hospital, Helsinki, Finland for their contribution in the operations reported in this study.

No benefits in any form have been received or will be received by a commercial party related directly or indirectly to the subject of this article. 


\section{References}

1. Kirkley A, Griffin S, McLintock H, Ng L. The development and evaluation of a diseasespecific quality of life measurement tool for shoulder instability: the Western Ontario Shoulder Instability Index (WOSI). Am J Sports Med 1998;26:764-72.

2. Hovelius L. Shoulder dislocation in Swedish ice hockey players. Am J Sports Med 1978;6:373-7.

3. McLoughlin HL, Cavallaro WU. Primary anterior dislocation of the shoulder. Am J Surg 1950;80:615-21.

4. Hovelius $\mathbf{L}$, Eriksson $\mathbf{K}$, Fredin $\mathbf{H}$, et al. Recurrences after initial dislocation of the shoulder. J Bone Joint Surg [Am] 1983;65-A:343-9.

5. Wintzell G, Haglund-Åkerlind Y, Tidermark J, Wredmark T, Eriksson E. A prospective controlled randomized study of arthroscopic lavage in acute primary anterior dislocation of the shoulder: one-year follow-up. Knee Surg Sports Traumatol Arthrosc 1996;4:43-

6. Torchia ME, Caspari RB, Asselmeier MA, Beach WR, Gavari M. Arthroscopic transglenoid multiple suture repair: 2 to 8 year results in 150 shoulders. Arthroscopy 1997;13:609-19.

7. Kandziora F, Jäger A, Bischof F, et al. Arthroscopic labrum refixation for post-traumatic anterior shoulder instability: suture anchor vs. transglenoid fixation technique. Arthroscopy 2000;16:359-66.

8. Kirkley A, Werstine R, Ratjek A, Griffin S. Prospective randomized clinical trial comparing the effectiveness of immediate arthroscopic stabilization versus immobilization and rehabilitation in first traumatic anterior dislocations of the shoulder: long-term evaluation. Arthroscopy 2005;21:55-63.

9. Baker CL, Uribe JW, Whitman C. Arthroscopic evaluation of acute initial shoulder dislocations. Am J Sports Med 1990;18:25-8.

10. Taylor DC, Arciero RA. Pathologic changes associated with shoulder dislocations: arthroscopic and physical examination findings in first-time, traumatic anterior dislocation. Am J Sports Med 1997;25:306-11.

11. Bigliani LU, Pollock RG, Soslowsky LJ, et al. Tensile properties of the inferior glenohumeral ligament. J Orthop Res 1992;10:187-97.

12. Caspari RB. Arthroscopic reconstruction for anterior shoulder instability. Techn Orthop 1988;3:59-66.

13. Steinbeck J, Jerosch J. Arthroscopic transglenoid stabilization versus open anchor suturing in traumatic anterior instability of the shoulder. Am J Sports Med 1998;26:373-8.

14. Okmura K, Takiuchi T, Aoki M, Ishii S. Labral shape after arthroscopic Bankart repair: comparisons between the anchor and Caspari methods. Arthroscopy 2005;21:194-9.

15. Yoneda M, Hayashida K, Izawa K, Shimada K, Shino K. A simple and secure anchoring system for Caspari's transglenoid multiple suture technique using a biodegradable poly--lactic acid button. Arthroscopy 1996;12:293-9.
16. Mologne TS, Lapoint JM, Morin WD. Arthoscopic anterior labral reconstruction using a transglenoid suture technique: results in active-duty military patients. $A m \mathrm{~J}$ Sports Med 1996;24:268-74.

17. Hobby J, Griffin D, Dunbar M, Boileau P. Is arthroscopic surgery for stabilisation of chronic shoulder instability as effective as open surgery? A systematic review and meta-analysis of 62 studies including 3044 arthroscopic operations. J Bone Joint Surg [Br] 2007;89-B:1188-96

18. No authors listed. Classified military information. http://www.mil.fi

19. World Health Organization. International classification of diseases. http:// www.who.int/classifications/icd/en (date last accessed 4 January 2008).

20. Kirkley A, Griffin S, Richards C, Miniaci A, Mohtadi N. A prospective randomized clinical trial comparing the effectiveness of immediate arthroscopic stabilization versus immobilization and rehabilitation in first traumatic anterior dislocations of the shoulder. Arthroscopy 1999;15:507-14.

21. Robinson CM, Dobson RJ. Anterior instability of the shoulder after trauma. J Bone Joint Surg [Br] 2004;86-B:469-79.

22. Barber FA, Ryu RK. Tauro JC. Should first-time anterior shoulder dislocations be surgically stabilized? Arthroscopy 2003;19:305-9.

23. Balg F, Boileau $\mathbf{P}$. The instability severity index score: a simple pre-operative score to select patients for arthroscopic or open shoulder stabilisation. J Bone Joint Surg [Br] 2007;89-B:1470-7.

24. van Oostveen DP, Schild FJ, van Haeff MJ, Saris DB. Suture anchors are superior to transglenoid sutures in arthroscopic shoulder stabilization. Arthroscopy 2006;22:1290-7.

25. Green MR, Christensen KP. Arthroscopic Bankart procedure: two- to five-year follow-up with clinical correlation to severity of glenoid labral lesion. Am J Sports Med 1995;23:276-81.

26. Savoie FH 3rd, Miller CD, Field LD. Arthroscopic reconstruction of traumatic anterior instability of the shoulder: the Caspari technique. Arthroscopy 1997;13:201-9.

27. Barber FA, Click JN. The effect of inflammatory synovial fluid on the breaking strength of new "long-lasting" absorbable sutures. Arthroscopy 1992;8:437-41.

28. Kagaya K, Yoneda M, Hayashida K, et al. Modified Caspari technique for traumatic anterior shoulder instability: comparison of absorbably sutures versus absorbable plus nonabsorbable sutures. Arthroscopy 1999;15:400-7.

29. Mazzocca AD, Brown FM Jr, Carreira DS, Hayden J, Romeo AA. Arthroscopic anterior shoulder stabilization of collision and contact athletes. Am J Sports Med 2005:33:52-60.

30. Ide J, Maeda S, Takagi K. Sports activity after arthroscopic superior labral repair using suture anchors in overhead-throwing athletes. Am J Sports Med 2005;33:507- 